20. POSITIONS AND MOTIONS OF MINOR PLANETS, COMETS AND SATELLITES

(POSITIONS ET MOUVEMENTS DES PETITES PLANETES, DES COMETES ET DES SATELLITES)

PRESIDENT: B. G. Marsden.

VICE-PRESIDENT: G. Sitarski.

ORGANIZING COMMITTEE: F. K. Edmondson, S. Ferraz-Me11o, P. Herget, E. I. Kazimirchak-Polonskaya, L. Kresák, W. H. Robertson, E. Roemer, J. Schubart, G. E. Taylor.

\title{
Introduction
}

The past triennium has been noteworthy for several significant discoveries in the outer part of the solar system. The discovery of the rings of Uranus was inspired by the activities of Commission 20's new Working Group on Occultations. Although the discovery of a probable Plutonian satelite has not yet been completely confirmed, the implication that Pluto's mass is only 0.002 earth masses may make it desirable to consider whether Pluto should be relegated to minorplanet status. The orbit of Pluto is in fact not greatly dissimilar to that of the object classified as the new minor planet (2060) Chiron, which was found to have its aphelion near the orbit of Uranus and its perihelion just inside the orbit of Saturn.

With the retirement of $\mathrm{P}$. Herget, who directed the IAU Minor Planet Center ever since its establishment at the Cincinnati Observatory in 1947, an era has come to an end. A pioneer in the use of punched-card machines in astronomy, Herget revolutionized the process of computation of special perturbations and orbit improvements. His influence in promoting an improvement in the quality of astrometric, orbital and ephemeris data on minor planets has been tremendous, as he anticipated the more stringent needs of those involved in making physical observations and in planning possible space missions to these objects.

In the observational area, it is greatly to be regretted that the program conducted by $\mathrm{E}$. Roemer at the University of Arizona was suspended at the end of 1976. The cessation of this program has had a very obvious effect on the lengths of the observed arcs of faint comets and minor planets, and as a result the future recovery of several of the newly-discovered short-period comets and Apollo objects will take greater effort. The most extensive of the currently active programs on faint objects is that under the direction of R. E. McCrosky at the Agassiz Station of the Harvard College Observatory, although particularly valuable contributions are also being made by $C$. Kowal and H.-E. Schuster with the large Schmidt telescopes at Palomar and the European Southern Observatory, respectively.

The sections of this report on Comets, Satellites and Prediction of 0ccultations have been prepared by Roemer, S. Ferraz-Mello and G. E. Taylor, respectively, although some condensation has been necessary in order to fit the allotted space. Contributions by several members and consultants, notably E. I. KazimirchakPolonskaya, V. A. Shor and N. Yakhontova-Samojlova, are gratefully acknowledged. Astronomy and Astrophysics Abstracts numbers are generally used for the references.

Among international meetings of interest to the Commission were IAU Colloquium No. 39, 'Relationships between Comets, Minor Planets and Meteorites', held in Lyons, France, in August 1976; IAU Colloquium No. 41, 'Dynamics of Planets and Satellites and Theories of Their Motion', Cambridge, England, August 1976; and IAU Symposium No. 81, 'Dynamics of the Solar System', Tokyo, Japan, May 1978. The proceedings of IAU Colloq. No. 39 have been published $(20.012 .049)$, as have those 
of IAU Collog. No. 25, 'The Study of Comets', Greenbelt, Maryland, October 1974 (18.012.014), and the invited papers at IAU Colloq. No. 28, 'Planetary Satellites', Ithaca, New York, August 1974, have also appeared $(20.012 .055)$.

Commission 20 notes with regret the passing of the following members: H. G. Hertz (1915-1976), H. M. Jeffers (1893-1976), A. Schmitt (1907-1975) and V. V. Michkovitch (1892-1976).

\section{Minor Planets}

GENERAL

By the time of Herget's retirement 4390 Minor Planet Circulars had been issued, 482 of them since the start of this triennium. The machine-readable file of observations contained some 190000 entries. On 1 July 1978 the Minor Planet Center moved to the Smithsonian Astrophysical Observatory under the direction of B. G. Marsden. C. M. Bardwell, who assisted in the minor-planet work in Cincinnati since 1958, moved to Cambridge with the Center, thereby assuring that continuity would be maintained. The Minor Planet Circulars are now being published on a regular monthly basis, and the last issue of 1978 was No. 4582 .

The Institute for Theoretical Astronomy in Leningrad, now under the direction of S. S. Lavrov, has continued to produce the annual volume Efemeridy Matykh Planet. Following the Recommendation discussed at the Grenoble meetings of Commission 20 the extended ephemerides in the 1978 edition incorporated the opposition effect, and the limiting mean-opposition magnitude for which these extended ephemerides are supplied was increased to 12.0 in 1978 and to 12.5 in 1979 . The list of unusual objects for which special extended ephemerides are provided has been extended to 26 entries, including all numbered minor planets with perihelion distances of less than $1.3 \mathrm{AU}$ - except for the lost object (719) Albert. The 1979 extended ephemerides give right ascension to 0.01 and declination to $0 ! 1$, while in the ordinary ephemerides the mean anomaly at the epoch is now given to $0: 1$. The new orbital elements introduced in the 1979 volume have been uniformly given for the standard osculation epoch JD 2444200.5 .

Among the several recent review articles on minor planets that by $\mathrm{C}$. $\mathrm{R}$. Chapman, J. G. Williams and W. K. Hartmann (Ann. Rev. Astron. Astrophys. 16, pp. 33-75) contains an extensive section (by Williams) on dynamics and orbital evolution, including discussions on Kirkwood gaps, Hirayama families, secular resonances and the Apollo and Amor objects.

\section{NEW RESULTS}

The most unusual discovery this triennium has been that by Kowal (20.098.061; IAU Symp. No. 81) of (2060) Chiron $=1977$ UB at almost the distance of Uranus. Prediscovery observations were subsequently noted near the times of the object's perihelion passages in 1945 and 1895. At the opposite extreme, the triennium also yielded the discoveries of the first three minor planets known to have orbits smaller than that of the earth: (2062) Aten = $1976 \mathrm{AA}, 1976 \mathrm{UA}$ and (2100) RaShalom $=1978 \mathrm{RA}$ were all recognized in the course of the Apollo-search program conducted by E. Helin and her associates with the 46- cm Palomar Schmidt, although $1976 \mathrm{UA}$ had several independent discoverers. Five other new Apollo objects were discovered, of which one, $1978 \mathrm{SB}$, has an orbit showing a remarkable resemblance to that of $\mathrm{P} /$ Encke. Recoveries of Apollo objects are now sometimes almost a matter of routine, and no fewer than 14 of the 27 known or suspected Apollos have been observed in more than one year.

Following earlier successes with other Apollo and Amor objects, (1580) Betulia was detected by radar at Arecibo Observatory during its favorable apparition in 1976, and signals were reflected from (1) Ceres early in 1977 (Bulz. Am. Astron. Soc. 9, p. 502; Ann. Rev. Astron. Astrophys. 16, pp. 265-292). 


\section{OBSERVATIONS AND ORBITS}

The program directed by N. S. Chernykh at the Crimean Astrophysical Observatory continues to be by far the most comprehensive one of its type, and during the triennium almost 7500 observations have been made of some 1500 numbered and more than 2800 unnumbered objects, many of them as faint as magnitude 18.0. At the Institute for Theoretical Astronomy G. R. Kastel' has computed preliminary orbits for more than 100 of the new objects, while similar computations - also for other one-opposition minor planets - have been made by Bardwell and Marsden at the Minor Planet Center.

In accordance with the Grenoble Resolution by Commissions 8 and 20 the termination date of V. I. Orel'skaya's program of observations of selected minor planets for star-catalogue improvement has been extended from 1975 to 1990. The earlier observations of the original ten minor planets have been combined into normal places and corrected to the FK4 system. The extended program, involving a total of 20 minor planets (18.098.041) and participation by over 30 observatories, has already yielded more than 5000 observations, of which 2000 have been processed. R. L. Branham (Astron. J. 83, pp. 675-681) has studied the orbits of five minor planets to determine corrections to the FK4 equator and equinox.

Some 30 observatories are also regularly involved in obtaining positions of substantial numbers of faint minor planets. Useful astrometric observations are a1so increasingly being made by amateur astronomers, notably in Japan and Germany, and also in England and Italy. The statement in the 1976 report that the longstanding Johannesburg program had been terminated was premature, but it now appears that the 1978 Johannesburg observations will indeed be the last. Accurate positions are still being measured when necessary from the older plates in the valuable collections at the Goethe Link, Lowell and Turku Observatories, and useful data could also come from the older Johannesburg plates.

As a by-product of his mass determinations for (1) Ceres and (2) Pallas, I. Schubart (18.098.034) published improved normal positions for the early observations of these objects.

At the Institute for Theoretical Astronomy F. B. Khanina and others have made accurate improvements of more than 320 sets of orbital elements of numbered minor planets during the triennium. A further 109 orbits have been improved under the guidance of M. A. Dirikis at the Latvian State University. Many of the 400 definitive orbits calculated by Herget in recent years have now been published. During the triennium Bardwell has computed orbits for more than 150 newly-numbered and soon-to-be-numbered minor planets, and some three dozen orbits of previously numbered and newly-numbered objects have been determined by Marsden. A few definitive orbits have been determined by L. D. Schmadel (Heidelberg), notably one that led to the recovery of the lost planet (1206) Numerowia (19.098.072), by D. K. Yeomans (Pasadena), by A. I. Vasyanovich (Tomsk), by G. M. Bocsa (Bucharest), and by R. Dvorak and C. Edelman (17.098.012). G. Sitarski was able to obtain a satisfactory orbit linking the 1936 and 1977 observations of Adonis (in spite of a close approach to Venus in 1964), which as of the end of 1978 was the latest numbered minor planet (2101). Also by the end of 1978 the number of numbered minor planets considered as more-or-less hopelessly lost had been reduced to only 23 .

The triennium has seen renewed interest in the establishment of identifications, and, in addition to some of those already mentioned, E. Bowell (Flagstaff), O. Kippes (Glattbach), Williams (Pasadena) and the Japanese amateurs H. Oishi and T. Urata have made important contributions in this area. Herget is preparing to extend the identification process with the use of a machine-readable file of the dates and positions of the centers of photographic plates in the collections at various observatories, and $\mathrm{S}$. J. Bus has been successful in locating several useful previously unrecognized images on old Palomar plates. 
T. Gehrels and N. Gehrels have prepared the fourth list of standard absolute magnitudes, extending up to (2082). The phase and opposition effects are defined as before, although the authors suggest that the fifth list should include individual phase coefficients when these are available.

\section{THEORETICAL INVESTIGATIONS}

Schubart (IAU Co1loq. No. 41; IAU Symp. No. 81) has continued his studies on commensurable motions of minor planets, with consideration in particular of the three-dimensional case; his suggestion that high-inclination Hilda-type ( $3: 2$ commensurability with Jupiter) and Thule-type (4:3) objects might exist led to searches for them by Schuster and R. M. West at the European Southern Observatory, but no such objects have yet been found. A study by C. Froeschlé and H. Scholl on the depletion of the outer part of the minor-planet belt also points to the need to search for objects in orbits of high inclination. In other recent papers $(14.098 .019 ; 17.098 .016 ; 19.042 .045 ; 20.098 .102$; IAU Symp. No. 81) these same authors have extended their earlier studies on commensurable motions and the Kirkwood gaps. W.-H. Ip $(17.098 .071 ; 18.098 .035 ; 20.098 .012 ; 20.098 .035)$ has a1so examined the $3: 2$ comensurability and the more general problem of collisions among minor planets. Investigations on orbits near the $2: 1$ resonance have been made by I. A. Gerasimov $(17.098 .049 ; 19.098 .022)$, by E. A. Grebenikov, O. A. Belousova and others $(18.098 .009 ; 18.098 .014)$, by S. Kasperczuk $(18.098 .017)$ and by Dirikis (Byulz. Inst. Teor. Astron. 14, No. 7).

R. Pratap (20.098.048; 20.098.049) has made a statistical and a kinematic theory of the Kirkwood gaps. T. Kiang (Nature 273, pp. 734-736; IAU Symp. No. 81), arguing strictly on the basis of the planar restricted problem, claims to have solved the matter of the gaps by demonstrating differences between the 2:1 and $3: 2$ resonances, but S. Aoki (Nature 275, p. 568) regards the question as still open.

R. Bien (19.042.022; Astron. Astrophys. 68, pp. 295-301) has examined the long-period effects in Trojan ( $1: 1$ comnensurability) motion, and B. Garfinkel (18.042.086; 19.042.051; 20.098.011; Celes. Mech. 18, pp. 259-275) has constructed a formal analytical long-period solution to the Trojan problem by the canonical Lie-Hori method. B. Erdi (Celes. Mech. 18, pp. 141-161) considers some aspects of Trojan motion in three dimensions, V. Banfi (17.098.083) studies the stability of large oscillations of Trojans around the libration points, and Gehrels (20.098.105) discusses the asymmetry of the preceding and following groups of Trojans.

Y. Kozai (IAU Symp. No. 81) has refined his 1962 work on secular perturbations on minor planets and the implications for the study of the Hirayama families; he also discusses further the question of argument-of-perihelion libration, suggesting that it takes place in the case of (944) Hidalgo. E. P. Aksenov and his colleagues (Moscow) are also investigating secular perturbations.

A. Carusi and E. Massaro (20.098.106) have made a statistical study of the families. 0 . Hansen $(20.098 .026)$ correlates dynamical and physical data on several families, while J. Gradie and B. Zellner (20.098.023) remark on observational evidence for the common origin of the members of selected families.

A paper by Shor and the late G. A. Chebotarev on the structure of the minorplanet belt (18.098.005) has been brought up to date (Fund. Cosmic Phys. 3, pp. 87-138). L. Kresák (19.098.013) estimates the total mass of the minor planets as $(3.0 \pm 0.5) \times 10^{21} \mathrm{~kg}$ and discusses the three-dimensional distribution of minor planets and comets (IAU Symp. No. 81). He also concludes (BuZZ. Astron. Inat. Czech. 29, pp. 149-154) that G. W. Wetherill (18.105.011) has probably overestimated the number of Apollo objects.

H. Hurnik (Poznañ) reports that he intends to continue the analytical computation of the perturbational function on minor planets by Brown's method. 
$\underline{\text { Comets }}$

GENERAL

The annual reports on comets for the years 1974 and 1975, including descriptive observational summaries, notes of comets searched for but not found, and tables of recently computed orbital elements, have been published by Marsden and Roemer (Quart. J. Roy. Astron. Soc. 19, pp. 38-58; ibia. 19, pp. 59-89).

With the transfer of the IAU Minor Planet Center to the Smithsonian Astrophysical Observatory less time-urgent material on comets is now appearing in the Minor Planet Circulars (or Minor Planets and Comets). Included are older astrometric observations, improved orbits and advance ephemerides. Urgent information continues to be transmitted by telegram and to appear in the airmailed IAU Circulars. The Kiev comet group provides a parallel service, with information appearing in the Kiev Kometnye TsirkuZyary; editorial responsibilities were borne by $\mathrm{S}$. $\mathrm{K}$. Vsekhsvyatskij in 1976-77 and jointly with K. I. Churyumov in 1978 .

\section{DISCOVERIES, RECOVERIES AND ASTROMETRIC OBSERVATIONS}

Comet activity continued at a high level during the 1976-78 triennium; for example, the total number of comets receiving designations during 1977 was a record 19, not counting one object that later proved to be a Pallas-type minor planet. Of the 15 newly discovered near-parabolic comets, eight were found visually by amateurs. Of the nine newly discovered short-period comets, all but two were found photographically by professionals. This statistical pattern is consistent with experience of recent decades. No designations were given to three comets found on plates being studied long after the time of observation.

One of the new short-period comets turned out to be a reappearance of the lost comet P/Denning 1 (1881 V), now designated P/Denning-Fujikawa (1978n). Three short-period comets were recovered at their first predicted returns to perihelion: $\mathrm{P} /$ Klemola (1976j), which had been observed for only six weeks in 1965, was found by G. Sause (Haute Provence); although the perihelion time in 1976 was thought to be uncertain by as much as two months, the required correction to the prediction was only -10.2 days. P/Kojima $(1977 \mathrm{r}$ ) was recovered by $\mathrm{H}$. Kosai and H. Hurukawa (using the 105-cm Schmidt at Tokyo's Kiso Station), and P/Clark (1978g) was found by T. Seki (Geisei, Japan), only a small correction being required to the prediction in each case. Kowal recovered $\mathrm{P} / \mathrm{Taylor}(1916 \mathrm{I}=1977 \mathrm{a})$, which had not been seen since the discovery apparition. The position was surprisingly close to that predicted by N. A. Belyaev and V. V. Emel'yanenko for the component B, which is now believed to be the principal component. After several unsuccessful attempts, Kowal recovered $P / J a c k s o n-N e u j m i n ~(1978 q)$, which had been lost from its discovery in 1936 until Kowal found it in 1970. Of the more routine recoveries, five were made by Roemer in 1976 and eight by McCrosky et al. in 1977-78. Seki made three further recoveries, while Z. M. Pereyra and his colleagues (Bosque Alegre) made two. P/Grigg-Skjellerup (1977b) was picked up by P. Jekabsons (Perth) and independently by A. C. Gilmore and P. M. Kilmartin (Wellington).

Four new comets of large perihelion distance were among the near-parabolic comets discovered during the triennium. Among them is the new record holder, comet Schuster $(1976 \mathrm{c}=1975 \mathrm{II})$, which has a perihelion distance of $6.88 \mathrm{AU}$.

Some 65 observatories have contributed to the astrometric work on comets during the triennium. The overwhelming majority of these observatories are located in the northern hemisphere. About 35 of the contributing observatories have fairly substantial programs, but the bulk of all astrometric observations continue to come from no more than 15 observatories. The southern hemisphere is underrepresented by a factor of two in number of programs, but this is to some degree compensated by outstanding effectiveness of the work of several participants; the contribution of the amateur D. Herald (Canberra) is particularly useful. 


\section{ORBITS AND ORBITAL EVOLUTION OF SHORT-PERIOD COMETS}

With powerful computing machinery now readily available, the standards of precision for prediction of returns of well-observed short-period comets are now very high. The overwhelming majority of ephemerides include compensation for all sensible planetary perturbations, as well as some form of allowance for nongravitational effects on comets observed at several perihelion passages.

Predictions for returning periodic comets continue to be published regularly, many of them in the annual Handb. Br. Astron. Assoc. Contributors included Marsden, Z. Sekanina, Yeomans, Sitarski, L. M. Belous, N. A. Bokhan, Yu. A. Chernetenko, Yu. V. Evdokimov, Kazimirchak-Polonskaya, S. Nakano, G. Schrutka, and several workers of the Computing Section of the British Astronomical Association. of those comets predicted to return to perihelion but not observed, most were poorly placed near the time of perihelion passage and not expected to be sufficiently bright while in observable position. $\mathrm{P} /$ Westphal, due at perihelion around the end of 1975, was not found in spite of extensive searches. There had been serious difficulties in linking the observations in 1852-53 with those in 1913, and the comet had faded out as it approached perihelion in 1913. P/van Houten, due at perihelion early in 1977, also was not recovered in spite of an extensive search by Kowal. The observed arc in 1960 had been less than five weeks, leaving an uncertainty of several months in the perihelion time.

Calculations of definitive osculating orbits for recently discovered periodic comets now normally include a least-squares adjustment of the elements to obtain a best fit to all the individual observations after an appropriate allowance for the effects of planetary perturbations. Among those who have participated in this phase of the work on comets during the triennium are Marsden (IAU Circulars, Minor Planet Circulars), R. J. Buckley (20.103.001), Kazimirchak-Polonskaya (ProbZ. Issled. Vselen. 7, pp. 340-383) and Nakano (Nakano wa Kangaeru Noda). Investigations have often included studies of the motions of recently discovered comets over a number of revolutions into the past and future. The most common finding is that a close approach to Jupiter has recently led to a substantial reduction in the perihelion distance, with the consequence that the comet can become brighter than previously. When this particular scenario does not apply, it is usually the case that the comet can only be observed when it reaches perihelion at certain times of the year, and if the period is close to an integral number of years for an extended stretch, the comet may repeatedly pass perihelion unobserved. It also appears that short-period comets are frequently experiencing substantial surges in brightness when they are first discovered.

Another group of studies involves the careful linking of the repeated returns to perihelion of wel1-observed short-period comets in order to investigate the orbital evolution of individual objects over times as long as a few centuries. Among them are: Emel'yanenko (19.103.802) on P/Tuttle; Evdokimov (19.103.231) on $\mathrm{P} /$ Brooks 2 and (20.103.211) on P/Giacobini-Zinner; Kazimirchak-Polonskaya (19.103. 472) and Belyaev and M. A. Merzlyakova (18.103.116) on P/Ashbrook-Jackson; Kazimirchak-Polonskaya $(17.103 .112 ; 19.103 .501)$ on P/Wolf; E. D. Kondrat'eva (Tmidy Astron. Obs. Kazan 44) on P/Crommelin; M. P. Imnadze (19.103.411) on P/Forbes; E. A. Reznikov (17.103.122) on P/Pons-Winnecke; and S. D. Shaporev (18.103.109; Probz. Kosmich. Fiz. 13, pp. 71-81) on P/de Vico-Swift. T. A. Vinogradova and Shor (Byulz. Inst. Teor. Astron. 14, No. 8) have determined new orbits for P/Brorsen-Metcalf in 1847 and 1919 and shown that nongravitational effects on the motion must have been substantially less than those found by Belous $(11.103 .117 ; 12.103 .102)$. Kazimirchak-Polonskaya (18.102.035) has reviewed the work carried out in the U.S.S.R. over the past 50 years on approaches of comets to Jupiter and the secular evolution of cometary orbits. Simulation studies made in general terms include that by H. Rickman and S. Vaghi (18.102.008), in which they examined the statistical characteristics of the transfer of comets between intermediate-period orbits and the observable part of the Jupiter family. They have 
also examined (19.102.024; Astron. Astrophys. Supp 2. 31, pp. 389-395) the distribution of Jovian perturbations on short-period comet orbits.

With the recovery during the past 15 years of a number of long-unobserved short-period comets, interest in this area continues high. Belyaev and his coworkers have refined and extended the work on single-apparition periodic comets summarized in the previous Commission 20 report. New work published during this triennium included the following: Belyaev and V. A. Izvekov (Byulz. Inst. Teor. Astron. 14, No. 6) on P/Denning 1 (1881 V; now P/Denning-Fujikawa $=1978 \mathrm{n})$, $\mathrm{P} /$ Barnard 1 (1884 II) and P/Brooks 1 (1886 IV); Belyaev and Emel'yanenko (Trudy Astron. Obs. Kazan 42) on P/Giacobini (1896 V), P/Metcalf (1906 VI), P/Schorr (1918 III) and P/du Toit 1 (1944 III = 1974 IV); and I. N. Murav'eva (Tmudy Astron. Obs. Kazan 42, 43) on P/Dubyago (1921 I) and P/Barnard 2 (1889 III). Buckley (19.102.009) has brought forward to the present time the elements of 11 short-period comets discovered between 1881 and 1918.

With interest in the comets of intermediate period stimulated by the expected return of $\mathrm{P} / \mathrm{Halley}$ in 1986 and the possibility of a space mission to this most active of periodic comets, Yeomans (19.103.191) has studied the motion over the interval 837-2061 and provided detailed ephemerides for the 1986 return. Some observations afmed at an early recovery have already been made. Yeomans finds that $\mathrm{P} / \mathrm{Ha}$ lley itself is well behaved, showing significant but stable nongravitational effects that are understandable in terms of loss of volatiles from a rotating icy nucleus. Other comets of similar period seem, however, to have erratic orbital motions ( $P /$ Brorsen-Metcalf and $P /$ Westphal), or to have nongravitational effects controlled by,ices more volatile than water-ice (P/Pons-Brooks and P/Olbers). Y.-C. Chang (Acta Astron. Sinica 19, pp. 109-118) also has studied the motion of $\mathrm{P} / \mathrm{Ha}$ lley, particularly as the basis for examination of historical records as far as three millennia into the past.

\section{ORBITS OF NEARLY PARABOLIC COMETS}

Marsden, Sekanina and E. Everhart (Astron. J. 83, pp. 64-71) have collected a sample of some 200 original orbits as basis for a detailed investigation of the "Oort effect" (the tendency for original $1 / a$ values to cluster within the very limited range from 0 to $+100 \times 10^{-6} \mathrm{AU}^{-1}$ ). Some 110 osculating orbits have been newly determined. This extends the 1973 work on comets of large perihelion distance, while recognizing that unknown nongravitational forces can be a problem with comets of smaller perihelion distance. An earlier version (20.102.044) included a historical overview of divergent ideas concerning the Oort cloud.

Work on the catalogue of one-apparition comets continues in Warsaw under the leadership of M. Bielicki, with the collaboration of Sitarski and K. Ziolkowski $(17.102 .013 ; 17.102 .036 ; 18.031 .282$; IAU Symp. No. 81). Some parts of the work are being carried out in Bratislava, through the cooperation of Kresák.

\section{THEORETICAL INVESTIGATIONS}

A variety of topics related to the Oort cloud and the origin of comets received attention during the triennium. I. Hasegawa (17.102.035) discussed the concentration of aphelia of long-period comets in the direction of the antapex of the solar motion. V. P. Tomanov $(17.102 .021 ; 17.102 .023 ; 19.102 .013 ; 20.102 .036)$ and V. V. Radzievskij and Tomanov (19.102.008; 20.102.002) have considered various aspects of the possible capture of comets upon passage of the sun through an interstellar cloud of planetesimals. Radzievskij and Tomanov (18.102.062), as well as I. Revina (19.102.016) and K. A. Shtejns and Revina (19.102.014), studied the influence of selection effects of discovery on the observed distribution of various orbital elements, including the concentration of perihelia.

Rickman (17.102.055), in his study of the significance for cometary capture of stellar perturbations on orbits of long-period comets, looked in particular at 
the dependence of the frequency, and relative importance over very long time intervals, of stellar passages as a function of the passage distance. P. D. Noerdlinger (19.102.002), who also examined the hypothesis of an interstellar origin of comets, considered the possibility of capture by the sun of particles from the low-energy portion of a population having a Maxwellian distribution at infinity. S. L. Piotrowski and Sitarski (17.102.032) analyzed the effect of mass accretion on orbital elements and discovery probability of a comet approaching the sun along a parabolic or slightly hyperbolic orbit. M. E. Bailey $(20.102 .027)$ reviewed in rather general terms the hypothesis of the Oort cloud, both as original$1 \mathrm{y}$ envisioned by Dort in 1950 and as discussed later by Lyttleton. Such differences between old and new comets as a relative prevalence of larger perihelion distances and an increased rate of post-perihelion fading for new comets are regarded by Kresák (20.102.020) as rather inconclusive because of statistical fluctuations in the observed population.

A. P. Boss and S. J. Peale $(17.102 .001)$ concluded that secular perturbations of long-period comets are more sensitive by several orders of magnitude for detection of an unseen cometary mass distribution at the fringes of the solar system than is precision tracking of deep space probes. E. M. Drobyshevskij (20.102.032; Astron. Tsirk. No. 962; Moon and Planets 18, pp. 145-194) considered the possible capture of comets from a hypothetical planetary-cometary cloud beyond Neptune's orbit by a mechanism depending in part on processes of accumulation in icy envelopes of planets. Everhart continued his studies of the processes by which shortperiod orbits evolve from near-parabolic ones. In recent work (IAU Symp. No. 81) he has been concerned with the relative shortage of comets in elliptical orbits having perihelion distance between 0 and 1 AU as compared with those in parabolic orbits with such perihelion distances. To resolve the problem it appears to be necessary to consider simultaneously the perturbations by the planets and by passing stars, as well as the dissipative effects that make a comet less observable on subsequent returns. P. R. Weissman (IAU Symp. No. 81) attempted to include all the effects in his study of the physical and dynamical evolution of comets.

Kresâk (BuZZ. Astron. Inst. Czech. 29, pp. 103-114; ibid. 29, pp. 114-125) has compiled a list of approaches of comets and minor planets to within $0.20 \mathrm{AU}$ of the earth as basis for a study of the population and size distribution of interplanetary bodies in the earth's environment. F. L. Whipple (19.102.010) has explored the reality of dynamical relationships among comet groups and concluded that, except in the case of the Kreutz sungrazers, the similarity of orbital elements within the groups is no greater than random expectation.

Sekanina has discussed the splitting of comets in a number of papers (19.102.003; 19.103.201; 20.102.042; Icarus 33, pp. 173-185; IAU Symp. No. 81). He finds that the motions of the fragments can be well modeled in a majority of cases by a formulation that depends only on the time of splitting and the difference between the effective solar attraction on the fragments, rather than on the impulse imparted at the time of splitting. A. Golubev $(14.102 .013 ; 18.102 .003)$ also studied disruptions of comets, concluding that comets subject to disruption are distinguished by greater relative brightness and high physical activity.

\section{Satellites}

\section{GENERAL}

Meetings on observations and ephemerides of planetary satellites have been held each year at the U.S. Naval observatory; the 1977 meeting coincided with the centennial of the discovery there of the satellites of Mars, and the proceedings of a special colloquium on these bodies (including some papers on astrometry and dynamics) are contained in Vistas Astron. 22, part 2. A symposium and workshop on the motions of natural and artificial satellites was held in Austin in December 1977; the proceedings will be published by the University of Texas Press. 
NEW AND UNCONFIRMED SATELLITES

The most spectacular event of the triennium has been the discovery of an elongation of Pluto, probably due to a satellite that has been temporarily designated $1978 \mathrm{P} 1$, by J. W. Christy with the $155-\mathrm{cm}$ astrometric reflector at the U.S. Naval Observatory's Flagstaff Station. The elongation was confirmed photographically at the Cerro Tololo and McDonald Observatories and visually at Mauna Kea (IAU Circ. Nos. 3241 and 3286; Astron. J. 83, pp. 1005-1008). The observations suggest a satellite 2-3 magnitudes fainter than Pluto in an orbit inclined at $105^{\circ}$ with respect to the plane of the sky. Seven approximate positions, including two separations, were obtained in 1978, as well as eight prediscovery observations in 1965, 1970 and 1977. The period is consistent with the lightcurve period of Pluto (6.3867 days), and the mean distance from the planet is about $20000 \mathrm{~km}$. L. Andersson (BuZZ. Am. Astron. Soc. 10, p. 586) remarks that a solution with the ascending (rather than the descending) node near $170^{\circ}$ fits the observations best and suggests that eclipse phenomena should occur during the next few years.

Analysis by J. W. Fountain and S. M. Larson of observations of faint images near Saturn appearing on exposures obtained with the Lunar and Planetary Laboratory's 154-cm reflector during the 1966 passage of the earth through the ring plane indicates the existence of another satellite of about the same brightness as A. Dollfus' satellite Janus and in a similar orbit; the objects are perhaps major members of an extended ring (Icarus 36, pp. 92-106). On the other hand, K. Aksnes and F. A. Franklin (ibid. 36, pp. 107-118) point out that, while the observations may suggest that satellites do exist near the rings (although the possibility of ring knots cannot be excluded), their orbits cannot be uniquely determined; they propose that Janus and the Fountain-Larson object be given the temporary designations $1966 \mathrm{~S} 1$ and $1966 \mathrm{~S} \mathrm{2.} \mathrm{Efforts} \mathrm{should} \mathrm{be} \mathrm{made} \mathrm{to} \mathrm{clarify} \mathrm{the}$ situation during the earth's 1979-80 ring-plane passage. Aksnes and Franklin also rediscuss the case of Pickering's 1904 satellite Themis and conclude that all evidence for its existence has vanished.

No additional observations have been reported of $1975 \mathrm{~J} 1$ (the probable fourteenth satellite of Jupiter). Attempts to recover it at McDonald and Palomar appear to have been unsuccessful.

\section{OBSERVATIONAL PROGRAMS AND TECHNIQUES}

Astrometric observations of the established satellites have been reported by 13 observatories. The program at McDonald included observations with some regularity of all the faint satellites of Jupiter ( $\mathrm{J}$ V-XIII) and of Saturn I-IX. G. F. Benedict, P. J. Shelus and J. D. Mulholland improved the techniques for plate measurement and reduction using a microdensitometer (18.031.297; 18.099.241; 19.099.509; Astron. J. 83, pp. 999-1002). Over 550 observations of the satellites of Mars, the Galilean satellites, Saturn II-VI and Saturn VIII were made at the Pulkovo Observatory by T. P. Kiseleva and others (20.100.031; ByuZZ. Inst. Teor. Astron. 14, No. 8). The Galilean satellites and Saturn I-VIII were also observed at the Leander McCormick Observatory by P. A. Ianna and P. Seitzer, who obtained more than 400 exposures at the 1976-77 and 1977-78 oppositions. Ianna also observed Uranus III and IV and Neptune I at Mount Stromlo. At Kazan some 400 positions of the Galilean and Saturnian satellites were obtained under the direction of I. Chugunov, and at Nikolaev about 100 plates were taken by V. Voronenko, G. Gore1' and F. Kalikhevich (20.041.049). Some 50 exposures of the satellites of Mars were obtained by D. Pascu at the U.S. Nava1 Observatory at the December 1975 and January 1978 oppositions, and the Pascu neutral-density-filter-spot technique was also used by R. L. Walker, Christy and R. S. Harrington to obtain positions of the satellites of Uranus and Neptune I (Astron. J. 83, pp. 838-844). Roemer obtained a few exposures of Jupiter XIII and Neptune II (Steward 229-cm reflector).

D. B. Campbel1 et al. (Icarus 34, pp. 254-267) have published Doppler shifts of the radar echoes from the Galilean satellites on 22 nights in 1975 and 1976. 
ORBITAL STUDIES AND THEORETICAL INVESTIGATIONS

Some 4900 observations of the satellites of Mars distributed at more than 20 oppositions were used by Shor $(14.042 .032 ; 18.097 .203)$ to determine new orbital elements, secular variations and the figure of Mars. A better representation of the observations is achieved as compared to earlier solutions. The acceleration of Phobos is found to be $\left(21: 4 \pm 22^{\circ}\right) \times 10^{-9}$ day $^{-2}$. The resulting longitudes represent well the positions derived from Mariner-9 TV data.

Radio-tracking data associated with the encounters between the satellites and the Viking Orbiter 1 spacecraft have been analyzed by $\mathrm{E}$. J. Christensen et $a \mathrm{l}$. (Geophys. Res. Lett. 4, pp. 555-557) and by R. H. Tolson et al. (ibia. 4, pp. 551$554)$. Their results are consistent with the combined value of $(1.63 \pm 0.12) \times$ $10^{-8}$ Mars masses for the mass of Phobos.

J. H. Lieske revitalized Sampson's theory for the Galilean satellites by removing algebraic errors, Introducing some neglected effects, allowing for nonzero-amplitude librations and providing final results as analytic functions of 49 arbitrary constants of integration and physical parameters (19.099.507). The series of photometric eclipses observed at Harvard from 1878 to 1903 has been combined with other post-1903 photometric eclipses in order to evaluate the parameters of the theory (Astron. Astrophys. 65, pp. 83-92). B. Brown (Celes. Mech. 16, pp. 229-259) removed short-period terms by Kamel's averaging technique and studied the long-period behavior of the orbits.

A comprehensive analysis by G. W. NulI (18.099.182) of Doppler data from Pioneer 10 and 11 during their encounters with Jupiter yielded improved values for the masses of the Galilean satellites and the harmonic coefficients of Jupiter. Theoretical investigations by Aksnes (IAU Colloq. No. 41), Ferraz-Mello (ibid.), E. N. Lemekhova (14.099.239) and J. L. Sagnier are in progress. An introductory book by Ferraz-Mello on the Dynamics of the Galilean Satellites is in press at the University of São Paulo.

Final analyses of the 1973 series of mutual phenomena of Jupiter's satellites have been published by Aksnes and Franklin (17.099.218) and by T. Nakamura (17.099.220). Aksnes and Franklin (Icams 34, pp. 188-193), and also J.-E. Arlot (Astron. Astrophys. Suppl. 34, pp. 195-197), have provided predictions for the mutual phenomena to occur in 1979.

From 31 available observations of Jupiter XIII covering nearly five revolutions about Jupiter, Aksnes (Astron. J. 83, pp. 1249-1256) determined a new orbit and computed an ephemeris from 1978 to 2000. T. V. Bordovitsyna and L. E. Bykova completed their numerical studies and determined new orbital elements for Jupiter VI-IX. New methods for solving ill-conditioned systems were used, and a new determination of the mass of Jupiter was made (Astron. Geod. Tomsk 6, pp. 32-41; ibid. 6, pp. 42-46; Byulz. Inst. Teor. Astron. 14, No. 7).

Y. Kozai (18.100.204) published an extension of his early work, with the masses of Saturn II, IV and VIII and the oblateness parameters of Saturn being recomputed using correct expressions for the forced eccentricity and mean longitude of S II and accurate expressions for the perturbations of the orbit of S VI due to S VIII. The results are $\mathrm{m}_{2}=(1.3 \pm 0.4) \times 10^{-7}, \mathrm{~m}_{4}=(1.85 \pm 0.04) \times$ $10^{-6}, \mathrm{~m}_{8}=(0.10 \pm 0.08) \times 10^{-5}$ Saturn masses. Also, the mass of $\mathrm{S} V, \mathrm{~m}_{5}=$ $(0.9 \pm 0.2) \times 10^{-5}$, is derived from the observed secular motions of six sate1lites. Y. Hatanaka (IAU Symp. No. 81) improved the orbital elements of S VIII. From observations taken in pairs since 1874, M. Rapaport (18.100.207) improved the mean motions of Saturn I-V. The values show agreement with Kozal's early work but not with the values proposed by H. A. Garcia (08.100.015). Rapaport also compared existing theories of S VIII with observations, finding it necessary to consider the resonance with S VI (Astron. Astrophys. 62, pp. 235-238). 
A. T. Sinclair (20.100.501) fitted recent photographic observations to existing theories of S III-VI and to his theory of S VIII (12.100.214) and derived improved orbital elements. From a gravitational model fitted to observed secular motions, W. I. McLaughlin and T. D. Talbot (19.100.020) determined $\mathrm{m}_{5}=(4.8 \pm$ $0.8) \times 10^{-6}$ and the mass of the ring $m_{R}=(6.2 \pm 2.4) \times 10^{-6}$ Saturn masses. A new theory of resonant satellite orbits developed by $W$. H. Jefferys and L. $M$. Ries has been applied to S II and S IV. With the participation of Mulholland they have now completed the discussion of observations for the determination of the constants of integration. L. Blitzer (Celes. Mech. 16, pp. 87-95) has applied spin-orbit theory to the $4: 3$ resonance between $S$ VI and $S$ VII.

A. W. Harris and C. F. Peters (19.100.504; 20.100.019), and also J. G. Porter and E. R. Delo (Handb. Br. Astron. Assoc. for 1977 and 1978; 20.100.512), provided predictions for two eclipses of S VIII by Saturn's rings in 1977-78. Aksnes and Franklin (Icams 34, pp. 194-207) have published predictions for the mutual phenomena of S I-VII during 1979-80.

R. Greenberg compared observations of Uranus $V$ with theory and obtained for the product of the masses of Uranus I and II an upper 1imit of about $10^{-9}$ Uranus masses $(18.101 .004)$. Uranus $V$ is $10^{\circ}$ ahead of the Iongitudes extrapolated from D. W. Dunham's elements, and this is the expected effect of U I and II. Dunham plans to update his orbital elements for the satellites of Uranus.

Ephemerides for Neptune II have been published yearly by Aksnes (17.101.006; 19.101.028; IAU Circ. No. 3219).

\section{Prediction of Occultations}

The Working Group on Prediction of Occultations was formed at the Commission 20 meetings in Grenoble, under the chairmanship of Taylor, to coordinate the rapidly increasing activity in this field and with the aim of providing a significant improvement in our knowledge of the shapes and sizes of satellites and minor planets. Twelve Buzletins giving details of predictions have been issued in the first two years of the Group's existence.

Orbits and astrometric ephemerides of selected minor planets (those whose diameters are believed to be greater than $100 \mathrm{~km}$ ) have been calculated by Shor and Dunham and supplied to Taylor, who has searched a combined AGK3 + SAO star catalogue against the ephemerides and issued predictions for any suitable occultations. Shor has also supplied orbits and ephemerides to Dunham and to D. Wallentinsen (an amateur in Albuquerque, New Mexico), while Dunham has also supplied ephemerides to the Lowell observatory. L. K. Kristensen and 0 . Moller have supplied Taylor with an ephemeris of (51) Nemausa.

The prediction service at Herstmonceux has been greatly expanded since it started in 1952, when predictions were issued for occultations by only the major planets and first four minor planets. At present, predictions involving catalogue stars are made for Mercury to Pluto, 16 satellites and about 50 minor planets. Plates are taken at Herstmonceux ahead of Saturn, Pluto and (2060) Chiron to enable occultations of faint stars to be detected. Similarly, plates taken at the Lick Observatory by A. R. Klemola have been used by Klemola, Marsden, W. Liller and J. L. Elliot to predict occultations of faint stars by Uranus and Neptune up to the end of $1980(19.101 .027 ; 20.101 .020 ; 20.101 .025 ; 20.101 .042)$. Shelus and Benedict have utilized the Palomar Sky Survey and microdensitometry techniques $(20.031 .209)$ to predict occultations.

The aim of producing preliminary predictions with a greater lead-time is gradually being achieved. Predictions of occultations by 39 minor planets for 1978 were issued in December 1977. Predictions for 49 minor planets for 1979 
were issued in August 1978, and it is hoped to issue predictions for $60-80$ minor planets for 1980 early in 1979. In addition, predictions of two special events have been issued further ahead for planning purposes: a possible occultation of a 12th-magnitude star by P1uto on 6 April 1980 and another of a 9 th-magnitude star by the rings of Uranus on 25 June 1985. Predictions for a number of events were circulated to amateur and professional observers throughout the world by varlous means, and these predictions were augmented by data issued by Dunham as President of the International Occultation Timing Association. The latter included predictions for a few events found by Wallentinsen.

These predictions, made so far ahead, are of necessity based solely on the ephemerides of the occulting bodies and on the catalogue positions of the stars. Accurate, reliable predictions can generally be made only when they are based on measurements of the occulting body and the star on the same plate. Normally this occurs only a few days before the event. Such observations have been made by L. H. Wassermann and O. G. Franz (Lowe11), W. S. Penhallow (University of Rhode Island), Klemola (Lick), Harrington (U.S. Naval Observatory), W. H. Robertson (Sydney), D. Harwood and M. P. Candy (Perth) and Taylor (Herstmonceux). Frenzied last-minute activity in developing and measuring plates and refining and distributing predictions by telephone and telex has finally led to several successful observations, notably the following:

5 March 1977: The brighter component (3rd magnitude) of the star $\gamma$ Ceti was occulted by (6) Hebe as seen by visual observers in Mexico. The observations were analyzed by Taylor and Dunham (Icarus 34, pp. 89-92), who obtained an equatorial diameter of $195 \pm 6 \mathrm{~km}$ and a polar diameter of $170 \pm 6 \mathrm{~km}$.

10 March 1977: The star SAO 158687 (magnitude 9) was occulted by Uranus. The original prediction by Taylor (10.101.001), based solely on the ephemerts of Uranus and the position of the star in the SAO catalogue, indicated that the occultation would be visible from all that part of the earth where Uranus would be visible in a dark sky. Results from plates taken at Perth and at Sydney two months before the occultation were consistent and indicated no occultation from anywhere on the earth. At the same time, results from plates taken at the lowell Observatory indicated a grazing occultation visible from part of the southern hemisphere (19.101.024). Although the on1y photoelectric observations of the main occultation were made from the Kuiper Airborne Observatory and at Cape Town, other occultations of the same star recorded at various observatories established the existence of a system of rings around the planet. A detailed analysis of the observations has been published by Elliot et al. (Astron. J. 83, pp. 980-992).

29 May 1978: The star AGK3 $+25^{\circ} 1763$ (magnitude 9) was occulted by (2) Pallas. Detailed planning and accurate last-minute predictions enabled successful photoelectric observations to be made from seven observatories. The main result is the determination of a mean equatorial diameter for Pallas of close to $540 \mathrm{~km}$.

7 June 1978: The star AGK3 $+4^{\circ} 1839$ (magnitude 6) was occulted by (532) Herculina. One photoelectric and two visual observations have enabled the determination of a diameter of about $220-230 \mathrm{~km}$ to be made. The observations also led to the discovery of an assumed satellite of Herculina (IAU Circ. No. 3241).

11 December 1978: The star AGK3 $+6^{\circ} 778$ (magnitude 8) was occulted by (18) Me1pomene. Photoelectric and visual observations in the Washington-Baltimore area showed that the star is double and yielded a preliminary value of $135 \mathrm{~km}$ for the diameter of the minor planet. A similar tracing recorded in Atlanta seems again to be attributable to an occultation by a satellite (IAU Circ. No. 3315).

\section{B. G. MARSDEN}

President of the Commission. 